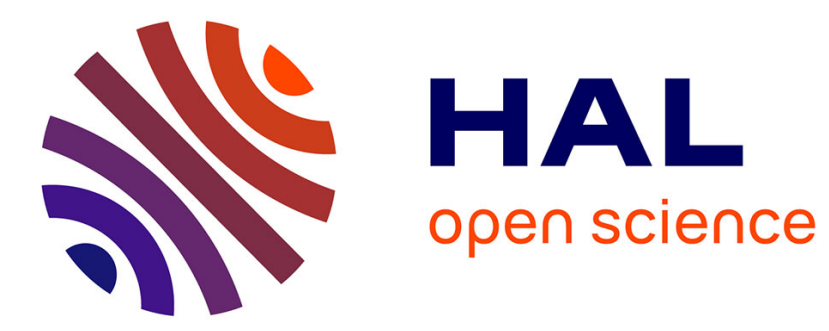

\title{
Disrupted iron storage in dental fluorosis
}

Sophia Houari, Emilie Picard, Tilmann Wurtz, Elsa Vennat, Nicolas Roubier, Ting Di Wu, Jean-Luc Guerquin-Kern, Mathieu Duttine, Tran Thu Thuy, Ariane Berdal, et al.

\section{> To cite this version:}

Sophia Houari, Emilie Picard, Tilmann Wurtz, Elsa Vennat, Nicolas Roubier, et al.. Disrupted iron storage in dental fluorosis. Journal of Dental Research, 2019, 98 (9), pp.994-1001. $10.1177 / 0022034519855650$. hal-02289423

\section{HAL Id: hal-02289423 \\ https://hal.sorbonne-universite.fr/hal-02289423}

Submitted on 16 Sep 2019

HAL is a multi-disciplinary open access archive for the deposit and dissemination of scientific research documents, whether they are published or not. The documents may come from teaching and research institutions in France or abroad, or from public or private research centers.
L'archive ouverte pluridisciplinaire HAL, est destinée au dépôt et à la diffusion de documents scientifiques de niveau recherche, publiés ou non, émanant des établissements d'enseignement et de recherche français ou étrangers, des laboratoires publics ou privés. 


\section{Disrupted iron storage in dental fluorosis}

Sophia Houari ${ }^{1,2}$, Emilie Picard $^{3}$, Tilmann Wurtz ${ }^{1}$, Elsa Vennat ${ }^{4}$, Nicolas Roubier $^{4}$, Ting Di Wu ${ }^{5,6}$,

Jean-Luc Guerquin-Kern ${ }^{5,6}$, Mathieu Duttine ${ }^{7}$, Tran Thu Thuy ${ }^{8}$, Ariane Berdal ${ }^{1,2,9}$ and Sylvie Babajko $^{1,2}$.

${ }^{1}$ Centre de Recherche des Cordeliers, INSERM UMRS 1138, Université Paris-Diderot, Sorbonne Université, Université Paris-Descartes, Laboratory of Molecular Oral Pathophysiology, F-75006 Paris, France.

${ }^{2}$ Université Paris-Diderot, Unit of Formation and Research (U.F.R.) Odontology, F-75006 Paris, France.

${ }^{3}$ Centre de Recherche des Cordeliers INSERM UMRS 1138 Université Paris-Diderot, Sorbonne Université, Université Paris-Descartes, from Physiopathology of Ocular Diseases to Clinical Development, F-75006 Paris, France.

${ }^{4}$ Laboratory of Mechanics of Soils, Structures and Materials (MSSMat), CNRS, Centrale-Supélec, Université Paris-Saclay, F-92290 Châtenay-Malabry, France.

${ }^{5}$ Institut Curie, INSERM U1196, Université Paris-Saclay, F-91405 Orsay, France.

${ }^{6}$ Université Paris-Sud, Université Paris-Saclay, CNRS UMR 9187, F-91405 Orsay, France.

${ }^{7}$ CNRS UPR 9048, Université de Bordeaux, Institute of Chemistry and Condensed Matter of Bordeaux, F-33600 Pessac, France.

${ }^{8}$ Faculty of Odonto-Stomatology, HochiMinh University of Medicine and Pharmacology, HôchiMinh Ville, Vietnam.

${ }^{9}$ Reference Center for Oral and Dental Rare Diseases (ORARES), Rothschild Hospital, F-75012 Paris, France. 
Corresponding author: Sylvie Babajko, 15 rue de l'Ecole de Médecine, Paris 75006 France.

Phone number: +331442755 79. E-mail address: sylviebabajko@ gmail.com

Abstract words: 249

Total words: 3545 (Abstract to Acknowledgment) - 3200 (Introduction to Discussion)

Total number of tables/figures: 5 figures

Number of references: 33

Key words: Ameloblasts; Dental Enamel; Fluorosis, Dental; Fluorides; Iron; Ferritins; Gene expression. 


\begin{abstract}
Enamel formation and quality are dependent on environmental conditions including exposure to fluoride which is a widespread natural element. Fluoride is routinely used to prevent caries. However, when absorbed in excess, fluoride may also lead to altered enamel structural properties associated to enamel gene expression modulations. As iron plays a determinant role in enamel quality, the aim of our study was to evaluate the iron metabolism in dental epithelial cells and forming enamel of mice exposed to fluoride, as well as its putative relation with enamel mechanical properties. Iron storage was investigated in dental epithelial cells using Perl's blue staining and SIMS imaging. Iron was mainly stored by maturation-stage ameloblasts involved in terminal enamel mineralization. Iron storage was drastically reduced by fluoride. Among the proteins involved in iron metabolism, ferritin heavy chain (Fth) in charge of iron storage, appeared as the preferential target of fluoride using RT-qPCR, western-blotting and immunohistochemistry analyses. Fluorotic enamel presented decreased quantity of iron oxydes as attested by ESR technique, altered mechanical properties measured by nanoindentation and ultrastructure analyzed by SEM/EDS. The in vivo functional role of Fth was illustrated using $\mathrm{Fth}^{+/-}$mice which incorporate less iron into their dental epithelium and exhibited poor enamel quality. These data demonstrate that exposure to excessive fluoride decreases ameloblast iron storage which contributes to the defective structural and mechanical properties in rodent fluorotic enamel. They raise the question of fluoride effects on iron storage in other cells and organs that may contribute to fluoride effects on population health.
\end{abstract}




\section{Introduction}

The human environment has become increasingly contaminated by various pollutants which may underlie the known modern burst of diseases (Gray et al. 2017). Fetal and perinatal exposure to some pollutants such as endocrine disruptors, dioxin, and fluoride generate irreversible enamel defects (Alaluusua et al. 2002; Aoba and Fejerskov 2002; Jedeon et al. 2013; Jedeon et al. 2016). Their characterization may help to reconstitute the history of ameloblast activity alterations and thus to use enamel defects as early marker of exposure to environmental toxicants altering the development of teeth and other organs. The present study is focused on fluoride which is widely present in human environment and which presents many cell effects (Liu et al. 2018) including disruption of iron metabolism (Niu et al. 2018). Fluoride protects against caries essentially via topical actions but, when absorbed in excess, fluoride may also lead to altered enamel structural properties associated to enamel gene expression modulations (Aoba and Fejerskov 2002; Fejerskov et al. 1994). As iron plays a determinant role in enamel quality (Dumont et al. 2014), as well as in cell activity and metabolism (Dixon and Stockwell 2014), the rationale of our study was to investigate the putative link that may exist between exposure to fluoride, disrupted iron metabolism in dental epithelial cells and enamel properties.

Enamel synthesis occurs from the third trimester of fetal life to six years after birth, a time-window particularly sensitive to environmental toxicants and fluoride (Bashash et al. 2017; Jedeon et al. 2013). Amelogenesis follows a well-known spatial-temporal sequence of ameloblast proliferation, differentiation, maturation and death, characterized by specific gene-expression patterns. Amelogenesis begins with the secretory stage, during which a partially mineralized enamel matrix is formed. Once the full thickness of the enamel matrix has been deposited, amelogenesis enters the maturation phase, during which enamel is fully mineralized by apatite crystal, reaching a 
mineral weight of approximately 97\% (Nanci 2012). Finally, ameloblasts disappear during tooth eruption, making enamel defects (if any) irreparable and thus irreversible, retrospectively recording ameloblast stresses. Thus, any disruption of ameloblast activity results in alterations of enamel structure.

Enamel can incorporate fluoride into the growing hydroxyapatite crystals, either by accretion or hetero-ionic substitution. Fluoride protects against caries by generating fluoridated apatite, increasing the remineralization process, and inhibiting bacterial enolase activity (Hamilton 1990; Moreno et al. 1974). However, limits to the prescription of fluoride have been repeatedly advised, mostly because of the narrow safety range for its use. The recommended dose to prevent caries $(0.05 \mathrm{mg} / \mathrm{kg} / \mathrm{day})$ is only half the amount that may cause enamel hypomineralization, called dental fluorosis (>0.1 mg/kg/day) (Fejerskov et al. 1994). Dental enamel which is constituted of hydroxyapatite is the most sensitive tissue due to fluoride high affinity for it (Aoba and Fejerskov 2002). In clinics, dental fluorosis is diagnosed by the presence of whitish opacities that may be associated with brownish coloration and enamel breakdown depending on the severity of the pathology. Enamel defects delineate the exposure window to fluoride. Mineralization-defects are comparable in fluorotic human teeth and fluorotic mouse incisors (Jalali et al. 2017).

In rodents, fluorotic enamel is characterized by discoloration of the physiological yellow-orange enamel pigmentation. Pigmented tooth enamel occurs not only in rodents, but also in several vertebrate clades, from mammals to fishes. Pigmented superficial enamel is harder and more resistant to acid and wear than core enamel, and this property is tightly associated with higher iron content (Bhattacharya et al. 2016; Gordon et al. 2015). In humans, trace elements, such as iron, may reinforce dental health (Dumont et al. 2014) and contribute to tooth resistance acquired during development. The most external part of enamel is in contact with maturation-stage ameloblasts 
which express and contain high concentrations of ferritin heavy chain (Fth) associated with the light chain (Ftl) to form the core subunit of ferritin, responsible for cellular iron storage (Wen and Paine 2013). Interestingly, ferritin expression has been reported regulated by fluoride in hepatocytes (Niu et al. 2018) suggesting a possible regulation in ameloblasts.

Ferritin, a 24-mer heteropolymer of Fth and Ftl subunits, plays a pivotal role in intracellular iron homeostasis, storing up free iron, hence preventing cellular free radical damage (Li et al. 2015). Fth has ferroxidase activity (converting $\mathrm{Fe}^{2+}$ to $\mathrm{Fe}^{3+}$ ). Ferric iron ions $\left(\mathrm{Fe}^{3+}\right)$ are transported and distributed throughout the body by transferrin (Trf) and internalized by cells mostly through its receptor Tfre, whereas ferrous iron ions $\left(\mathrm{Fe}^{2+}\right)$ are internalized through Divalent Metal Transporter 1 (Slc11a2) (Andrews 1999). The export of iron out of the cell is ensured by ferroportin (Slc40a1) (Donovan et al. 2005). The functions of these proteins have been demonstrated in vivo using mouse models. Among them, mice with total inactivation of Fth die before birth (Ferreira et al. 2000), but heterozygous $\mathrm{Fth}^{+/-}$mice are viable and may thus be a good model to study the functional role of Fth (Ferreira et al. 2001).

The main objective of the present study was to decipher the fluoride mechanism of action using the tooth enamel, its major target tissue. We tested the hypothesis of a disruption of iron storage in ameloblasts with putative alterations in enamel structural and mechanical properties, in an experimental model of dental fluorosis in mouse incisor. 


\section{Materials and Methods}

Complete procedures are presented in the appendix.

\section{Animals and biological samples}

Forty male Balb/c mice (aged three months) were used for this study and maintained in accordance with the French Ministry of Agriculture guidelines for the care and use of laboratory animals (number A75-06-12). This study adheres to the ARRIVE guidelines for animal research (Kilkenny et al. 2010). Wild type mice $(\mathrm{n}=20)$ and mice deleted for one allele of the Fth $\left(\mathrm{Fth}^{+/-}\right)(\mathrm{n}=20)$ were divided into two groups, treated or not with $5 \mathrm{mM} \mathrm{NaF}$ (equivalent to $100 \mathrm{ppm}$ ) in their drinking water for five weeks, the time necessary for full growth of the incisor (10 animals per group). Hemimandibles were fixed in 4\% PFA for nanoindentation, SEM/EDS, SIMS, Perl's staining, and immunofluorescence (IF) analyses. Right and left incisors were treated as separate biological samples but never used together in a same experiment. Three incisors from 3 different mice per group were used for nanoindentation and SEM/EDS, 3 for SIMS and 3 for IF and Perl's staining. Five and six hemimandibles were immediately microdissected for protein and RNA extractions, respectively.

\section{Perl's staining}

Incisor sagittal sections were deparaffinized, rehydrated, and immersed in $1 \mathrm{~N} \mathrm{HCl} / 5 \%$ potassium ferrocyanide for $40 \mathrm{~min}$. The slices were mounted with cover slips.

\section{Secondary Ion Mass Spectrometry (SIMS) imaging}


Ultra-thin frontal sections of incisors were deposited on silicon chips. Dynamic SIMS imaging was performed using a NanoSIMS-50 ion microprobe (CAMECA) operating in scanning mode (Guerquin-Kern et al. 2005).

\section{Electron Spin Resonance (ESR)}

Powders of dental enamel from the tips (erupted parts) of control and fluorotic mandibular incisors were prepared after careful grinding of the dentine with a diamond microbur and analyzed using a X-band ESR spectrometer.

\section{Nanoindentation}

Nanoindentation was performed on frontal sections of mouse mandibular incisors. The indentation experiments were performed using a calibrated Berkovich nanoindenter.

\section{Scanning Electron Microscopy (SEM) and Energy Dispersive X-ray Spectroscopy (EDS)}

SEM and EDS analyses of polished frontal sections of etched mouse mandibular incisors were performed with a Hitachi SU8030 microscope operating at $15 \mathrm{keV}$.

\section{RNA extraction and RT-qPCR analysis}

RNAs were extracted from microdissected incisor dental epithelium using the RNeasy® Protect Mini Kit (Qiagen). The detailed procedure of RT-qPCR is described in Appendix Materials file and primers listed in Appendix Table 1.

\section{Western blotting}


Total cellular proteins from freshly microdissected incisor enamel organs were extracted using a SDS-lysis buffer and were subjected to SDS-PAGE. Membranes were incubated with specific antibodies listed in Appendix Table 2.

\section{Immunofluorescence assays}

Sagittal sections of mouse hemimandibles were first incubated with antibodies directed against studied proteins, second with anti-IgG antibodies coupled to Alexa Fluor listed in Appendix Table 2. Finally, slices were immersed in DAPI and imaged with a Fluorescence microscope Zeiss Axiovert 200M.

\section{Statistics}

Results are expressed as the mean \pm SD and analyzed using Mann-Whitney's test with the GraphPad Prism Software Version 5.0 (GraphPad Software). 


\section{Results}

\section{Iron content imbalance in fluoride treated dental epithelia}

As iron is involved in enamel structural properties (Bhattacharya et al. 2016; Gordon et al. 2015) altered by excessive fluoride and, as ferritin expressing maturation-stage ameloblasts are the main target cells of fluoride (Aoba and Fejerskov 2002), we investigated the putative link between exposure to fluoride and disruption iron storage in maturation-stage ameloblasts. Under control conditions, Perl's staining showed mouse ameloblasts to contain an increasing amount of iron during the process of amelogenesis, from the transition- to maturation- and pigmentation-stages (Figure 1A and 1B) in accordance with previously published data (Miyazaki et al. 1998; Wen and Paine 2013). Chronic fluoride intake substantially decreased Perl's staining in maturation- and pigmentation-stage ameloblasts with a proximo-distal gradient (Figure 1C, encircled). The total length of the Perl's stained region, corresponding to iron-containing ameloblasts, was shorter and discontinuous with groups of ameloblasts partially or completely devoid of staining. There was also a loss of polarity and iron storage around some pigmentation-stage ameloblasts (Figure 1D, arrows).

A similar reduction of iron content was also observed using SIMS analysis (Figure 1F). This method allows the detection of iron as mass resolved ${ }^{56} \mathrm{Fe}^{16} \mathrm{O}^{-}$ions. Under control conditions, iron storage was in maturation-stage ameloblasts. In contrast, the signal corresponding to ${ }^{56} \mathrm{Fe}^{16} \mathrm{O}^{-}$in maturation-stage ameloblasts of NaF-treated mice was undetectable under the same measurement conditions whereas phosphorous, used as internal control, remained unchanged. 


\section{Alterations in paramagnetic species in fluorotic enamel}

ESR analysis of rodent enamel showed different signals mainly due to isolated $\mathrm{Fe}^{3+}$ paramagnetic ions and magnetic iron-bearing compounds. Iron oxides and/or hydroxides usually give broad ESR lines, from tenth of $\mathrm{mT}$ to hundreds of $\mathrm{mT}$ depending on their crystallinity and particle size (Figure $1 \mathrm{G})$. At room temperature $(293 \mathrm{~K})$, the X-band ESR spectrum of sound enamel mostly exhibited a broad and asymmetric resonance line at low magnetic fields $(\mathrm{g} \approx 8)$, and a narrower signal centered at $275 \mathrm{mT}(\mathrm{g} \approx 2.4)$ which may be due to small particles of iron oxides, magnetite $\left(\mathrm{Fe}_{3} \mathrm{O}_{4}\right)$ and hematite $\left(\mathrm{Fe}_{2} \mathrm{O}_{3}\right)$, respectively. At low temperature $(4.2 \mathrm{~K})$, the ESR spectrum revealed the presence of isolated $\mathrm{Fe}^{3+}$ ions located at two distinct sites with different symmetry $\left(\mathrm{C}_{2 \mathrm{v}}\right.$ for the $\mathrm{g}=$ 4.3 signal and $\mathrm{D}_{4 \mathrm{~h}}$ or $\mathrm{D}_{3 \mathrm{~d}}$ for the $\mathrm{g}=6.0$ line). Under fluoride treatment, there was a significant decrease in intensity for the signals detected at $\mathrm{g} \approx 8.0, \mathrm{~g} \approx 2.4$, and $\mathrm{g}=2.06$, corresponding mainly to iron-bearing secondary phases (oxides and oxyhydroxides).

\section{Expression modulations of genes involved in iron metabolism in fluoride treated dental epithelia}

We next investigated possible fluoride-mediated enamel alterations in the expression of genes involved in blood iron homeostasis (Hamp and Trf), entry (Tfrc and Slc11a2), storage (Fth and $\mathrm{Ftl}$ ), and export (Slc40a1) to explain the mechanisms of action of iron in enamel defects (Figure 2A-H). RT-qPCR analysis showed dental epithelial cells to express all tested mRNAs, except Trf mRNA (Figure 2A). Fluoride slightly increased Tfrc and Slc40a1 mRNA levels and reduced Fth mRNA level contrary to Ftl which wasn't significantly modulated. Fluoride also reduced Fth protein levels evaluated by immunofluorescence and western blotting (Figure 2E and 2G). Fth was mostly localized in maturation-stage ameloblasts (Figures 2B and 2E) which were the cells able to 
accumulate iron (Figure 1). The three proteins involved in transmembrane iron flux, Tfrc, Slc40a1, and Slc11a2, were not only present in ameloblasts but also in papillary layer (PL) cells (Figures 2C, 2D and 2F). Tfrc and Slc11a2 were localized to the basolateral side of ameloblasts (in front of PL cells), whereas Slc40a1 was localized to both the apical (in front of enamel) and basolateral sides, in contrast to other polarized epithelial cells that express Slc11a2 on the basolateral membrane only (Canonne-Hergaux et al. 1999). This represents the only example of polarized epithelial cells in which the iron export protein is present on both sides of the cell.

\section{Iron content imbalance in fluoride treated $\mathrm{Fth}^{+/-}$mice}

Fth being a target gene of fluoride, its involvement in amelogenesis was investigated using $\mathrm{Fth}^{+/-}$ mice (Ferreira et al. 2001). Late maturation- and pigmentation-stage ameloblasts presented a decreased ability to store iron compared to control mice as revealed by Perl's staining and SIMS imaging when one Fth allele was deleted (Figure 3A-C). NaF treatment reduced further iron content in ameloblasts similarly to control mice as well as the length of area containing labeled ameloblasts. Areas with groups of ameloblasts partially or completely devoid of staining were greater in $\mathrm{NaF}$ treated $\mathrm{Fth}^{+/-}$than controls (Figure 3A encircled). A similar reduction of iron content was also observed using SIMS analysis (Figure 3D).

In addition to the expected decrease in Fth expression in $\mathrm{Fth}^{+/-}$mice, $\mathrm{NaF}$ further decreased its expression at lower levels than in $\mathrm{NaF}$ treated control mice. The Tfre signal was stronger in NaFtreated $\mathrm{Fth}^{+/-}$mice, similar to the changes observed in NaF-treated wild type mice (Figure 3E). The Slc11a2 signals were reduced in mature ameloblasts, as well as in PL cells of $\mathrm{Fth}^{+/-}$mice and decreased further in $\mathrm{NaF}$-treated $\mathrm{Fth}^{+/-}$mice at lower levels than in NaF-treated control mice 
(Figure 3F). Slc40a1 levels were decreased in $\mathrm{Fth}^{+/}$mice at the RNA (Figure 3K) and protein (Figure 3L) level and compensated by the slight increased by NaF.

\section{Structural and mechanical alterations of fluorotic and Fth+/- teeth}

In order to understand the relation between disruption of iron content either upon exposure to fluoride or when one Fth allele was deleted. Fine observation of incisors showed that $\mathrm{Fth}^{+/-}$mice presented white enamel in absence of any treatment comparable to NaF treated mice (Figure 4A and 4B). More importantly, $\mathrm{Fth}^{+/-}$mice presented broken incisors when challenged by exposure to $\mathrm{NaF}$ (Figure 4B).

In order to explain this phenotype, control and $\mathrm{Fth}^{+/-}$incisors were investigated for their mechanical and ultrastructural properties using nanoindentation technique and SEM/EDS analyses respectively (Figure 4C-G). The mean values of the hardness and elastic moduli of sound enamel increased from the dentin enamel junction (DEJ) to the enamel surface for control mouse incisors with the highest values in the pigmented enamel (Figure 4C, Appendix Figure 1B, black line). There was a significant decrease in enamel hardness in $\mathrm{Fth}^{+/-}$mice compared to wild types especially for pigmented enamel (Figure 4C, black line vs dotted line). All the values of hardness and elastic moduli were clearly lower in fluorotic enamel (red lines) than in sound enamel (black lines) irrespective of the area studied.

SEM analysis showed that fluoride administration induced shorter and larger rods with many small irregularly shaped crystallites in rod enamel as well as in interrod enamel (Figure 4F).

In addition, SEM imaging showed dark areas in outer enamel (OE) of $\mathrm{Fth}^{+/-}$mice with larger crystallites, difficult to distinguish, they appeared as aggregates that resisted during etching (Figure 4E-F). EDS analysis revealed a significant decrease in $\mathrm{Ca} / \mathrm{P}$ ratio in enamel of $\mathrm{Fth}^{+/-}$mice whatever 
areas investigated (Figure $4 \mathrm{G}$ ). NaF treatment appeared to protect the crystallites of prismatic enamel of $\mathrm{Fth}^{+/-}$mice from acidic dissolution (Figure 4F). 


\section{Discussion}

We showed here that exposure to excessive fluoride reduces iron storage in ameloblasts and, iron amount in enamel. Fluoride affects several proteins involved in iron homeostasis, this suggests that the fluorosis phenotype might be mediated, at least in part, by a disruption in iron content in the ameloblasts. Rodent incisor enamel, unlike human has a high level of iron and this is incorporated directly into the crystal structure (Gordon et al. 2015; Dumont et al. 2014). We tested our hypothesis by treating Fth heterozygous mice with fluoride and assessed the extent of enamel damage. The results show that lowering levels of Fth further using genetic means, exacerbates the phenotype produced by $\mathrm{NaF}$ alone.

The consequence, clearly visible in rodents, is the whitish opacities on enamel attesting the suppression of iron deposition in the outer layer. Its mechanical consequence is the lowering of enamel hardness in accordance with studies showing that iron may improve enamel quality (Gordon et al. 2015; Bhattacharya et al. 2016). Nanoindentation method was used here, as it's the principal method to study the mechanical behavior and reliability of enamel. We found reduced Young's elastic moduli values and hardness of fluorotic enamel. This reduction in enamel hardness was also observed when one allele of Fth was deleted, especially in pigmented enamel, thus illustrating Fth and iron functional involvement in enamel mechanical properties. In addition, SEM and EDS analyses highlighted Fth involvement in enamel prismatic structure and mineralization process underlying the importance of iron in ameloblast activity. These results are in accordance with ESR spectroscopy which showed that iron oxides and oxyhydroxides appeared affected under fluoride treatment. Decreased iron content renders enamel prone to erosion and demineralization (Dumont et al. 2014). Here, we correlated the reduced iron content in fluorotic weakened enamel with a depletion of iron in enamel forming cells. Fluoride prevented the physiological storage of 
iron in ameloblasts by reducing and interrupting the length of the region containing colored ameloblasts, suggesting premature iron delivery and explaining the discoloration of fluorotic enamel. In addition to its multiple extracellular effects, including disruption of amelogenin assembly (Denbesten and Li 2011) and mineralization (Bronckers et al. 2009), fluoride also has intracellular effects through the modulation of gene expression, independently of the mineralization phase (Houari et al. 2014; Wurtz et al. 2008). Fluoride modulates the expression of many key genes involved in amelogenesis, such as the enamel matrix protein amelogenin and the proteolytic enzymes KLK-4 and MMP-20 (Li et al. 2018; Suzuki et al. 2014). In the present work, an analysis of the expression modulations of genes involved in iron storage and transportation revealed, for the first time, Fth as a fluoride target. Fluoride can thus influence iron homeostasis, via the reduced expression of Fth, and decreased amount of Fth is associated with weaken enamel quality. Moreover, the cellular localization of iron transporters showed that the remaining extracellular iron present at the apical side of ameloblasts was retaken up by the increased amount of Tfrc on the apical membrane of ameloblasts to compensate for its lower level. In Fth ${ }^{+/-}$mice, increase in Tfrc and decrease in Slc40a1 levels were expected as a feed-back loop regulation because of the lower expression of Fth. NaF treatment of $\mathrm{Fth}^{+/-}$mice enhanced these modulations, leading to higher iron re-uptake due to higher Tfrc levels. Thus, available iron for secretion into the enamel matrix was even more strongly reduced leading to colorless enamel (Figure 5). Finally, the well-known preferential effect of fluoride on maturation-stage ameloblasts may be due, at least in part, to their capacity to store iron.

All these data demonstrate the relation between exposure to excessive fluoride and the loss of iron in fluorotic enamel defects that may occur in rodents. We hypothesize a similar relation in humans, that needs to be further investigated, as iron present as trace element in human enamel has been 
reported to contribute to enamel structural properties with a lower relative content of carbonate type A (Ghadimi et al. 2013). In addition, only high-dose fluoride effects leading to fluorosis were investigated raising the question of fluoride low-dose effects.

More generally, these data raise the question of fluoride effects on iron storage in other cells and organs associated to oxidative stress that may contribute to fluoride effects on population health.

\section{Acknowledgments}

The University Paris-Diderot and the French National Institute of Health and Medical Research (INSERM) funded this work. We thank Sophia Loiodice and Christophe Klein (INSERM, Paris) for their technical help. We thank Ivan Moura (INSERM Paris) for providing $\mathrm{Fth}^{+/-}$mice and Zoubida Karim (CNRS, Paris) for the Ferroportin antibody. We acknowledge the PICT-IBiSA imaging facility at the Curie Institute for use of the ion microprobe (SIMS) and Sonia Prince at the CEF platform for the animal care (INSERM, Paris).

The authors declare they have no actual or potential competing financial interests nor any conflicts. 


\section{References}

Alaluusua S, Kiviranta H, Leppaniemi A, Holtta P, Lukinmaa PL, Lope L, Jarvenpaa AL, Renlund M, Toppari J, Virtanen H et al. 2002. Natal and neonatal teeth in relation to environmental toxicants. Pediatric research. 52(5):652-655.

Andrews NC. 1999. The iron transporter dmt1. The international journal of biochemistry \& cell biology. 31(10):991-994.

Aoba T, Fejerskov O. 2002. Dental fluorosis: Chemistry and biology. Crit Rev Oral Biol M. 13(2):155-170.

Bashash M, Thomas D, Hu H, Martinez-Mier EA, Sanchez BN, Basu N, Peterson KE, Ettinger AS, Wright R, Zhang Z et al. 2017. Prenatal fluoride exposure and cognitive outcomes in children at 4 and 6-12 years of age in mexico. Environmental health perspectives. 125(9):097017.

Bhattacharya PT, Misra SR, Hussain M. 2016. Nutritional aspects of essential trace elements in oral health and disease: An extensive review. Scientifica. 2016:5464373.

Bronckers AL, Lyaruu DM, DenBesten PK. 2009. The impact of fluoride on ameloblasts and the mechanisms of enamel fluorosis. J Dent Res. 88(10):877-893.

Canonne-Hergaux F, Gruenheid S, Ponka P, Gros P. 1999. Cellular and subcellular localization of the nramp2 iron transporter in the intestinal brush border and regulation by dietary iron. Blood. 93(12):4406-4417.

Denbesten P, Li W. 2011. Chronic fluoride toxicity: Dental fluorosis. Monographs in oral science. 22:81-96.

Dixon SJ, Stockwell BR. 2014. The role of iron and reactive oxygen species in cell death. Nat Chem Biol. 10(1):9-17.

Donovan A, Lima CA, Pinkus JL, Pinkus GS, Zon LI, Robine S, Andrews NC. 2005. The iron exporter ferroportin/slc40a1 is essential for iron homeostasis. Cell metabolism. 1(3):191200.

Dumont M, Tutken T, Kostka A, Duarte MJ, Borodin S. 2014. Structural and functional characterization of enamel pigmentation in shrews. Journal of structural biology. 186(1):38-48.

Fejerskov O, Larsen MJ, Richards A, Baelum V. 1994. Dental tissue effects of fluoride. Advances in dental research. 8(1):15-31.

Ferreira C, Bucchini D, Martin ME, Levi S, Arosio P, Grandchamp B, Beaumont C. 2000. Early embryonic lethality of $\mathrm{h}$ ferritin gene deletion in mice. The Journal of biological chemistry. 275(5):3021-3024.

Ferreira C, Santambrogio P, Martin ME, Andrieu V, Feldmann G, Henin D, Beaumont C. 2001. $\mathrm{H}$ ferritin knockout mice: A model of hyperferritinemia in the absence of iron overload. Blood. 98(3):525-532.

Ghadimi E, Eimar H, Marelli B, Nazhat SN, Asgharian M, Vali H, Tamimi F. 2013. Trace elements can influence the physical properties of tooth enamel. Springerplus. 2:499.

Gordon LM, Cohen MJ, MacRenaris KW, Pasteris JD, Seda T, Joester D. 2015. Dental materials. Amorphous intergranular phases control the properties of rodent tooth enamel. Science. 347(6223):746-750. 
Gray JM, Rasanayagam S, Engel C, Rizzo J. 2017. State of the evidence 2017: An update on the connection between breast cancer and the environment. Environmental health : a global access science source. 16(1):94.

Guerquin-Kern JL, Wu TD, Quintana C, Croisy A. 2005. Progress in analytical imaging of the cell by dynamic secondary ion mass spectrometry (sims microscopy). Biochimica et biophysica acta. 1724(3):228-238.

Hamilton IR. 1990. Biochemical effects of fluoride on oral bacteria. J Dent Res. 69 Spec No:660667; discussion 682-663.

Houari S, Wurtz T, Ferbus D, Chateau D, Dessombz A, Berdal A, Babajko S. 2014. Asporin and the mineralization process in fluoride-treated rats. Journal of bone and mineral research : the official journal of the American Society for Bone and Mineral Research. 29(6):14461455.

Jalali R, Guy F, Ghazanfari S, Lyaruu D, van Ruijven L, DenBesten P, Martignon S, Castiblanco G, Bronckers A. 2017. Mineralization-defects are comparable in fluorotic impacted human teeth and fluorotic mouse incisors. Archives of oral biology. 83:214-221.

Jedeon K, De la Dure-Molla M, Brookes SJ, Loiodice S, Marciano C, Kirkham J, Canivenc-Lavier MC, Boudalia S, Berges R, Harada H et al. 2013. Enamel defects reflect perinatal exposure to bisphenol a. The American journal of pathology. 183(1):108-118.

Jedeon K, Houari S, Loiodice S, Thuy TT, Le Normand M, Berdal A, Babajko S. 2016. Chronic exposure to bisphenol a exacerbates dental fluorosis in growing rats. Journal of bone and mineral research : the official journal of the American Society for Bone and Mineral Research. 31(11):1955-1966.

Kilkenny C, Browne WJ, Cuthill IC, Emerson M, Altman DG. 2010. Improving bioscience research reporting: The arrive guidelines for reporting animal research. PLoS biology. 8(6):e1000412.

Li J, Wang P, Gao J, Fei X, Liu Y, Ruan J. 2018. Naf reduces klk4 gene expression by decreasing foxo1 in 1s8 cells. Biological trace element research.

Li W, Garringer HJ, Goodwin CB, Richine B, Acton A, VanDuyn N, Muhoberac BB, IrimiaDominguez J, Chan RJ, Peacock $M$ et al. 2015. Systemic and cerebral iron homeostasis in ferritin knock-out mice. PloS one. 10(1):e0117435.

Liu X, Nie ZW, Gao YY, Chen L, Yin SY, Zhang X, Hao C, Miao YL. 2018. Sodium fluoride disturbs DNA methylation of nnat and declines oocyte quality by impairing glucose transport in porcine oocytes. Environ Mol Mutagen. 59(3):223-233.

Moreno EC, Kresak M, Zahradnik RT. 1974. Fluoridated hydroxyapatite solubility and caries formation. Nature. 247(5435):64-65.

Nanci A. 2012. Ten cate's oral histology: Development, structure, and function. Ed.8. C.V. Mosby, maryland heights, mo.

Niu Q, He P, Xu S, Ma R, Ding Y, Mu L, Li S. 2018. Fluoride-induced iron overload contributes to hepatic oxidative damage in mouse and the protective role of grape seed proanthocyanidin extract. J Toxicol Sci. 43(5):311-319.

Suzuki M, Shin M, Simmer JP, Bartlett JD. 2014. Fluoride affects enamel protein content via tgfbeta1-mediated klk4 inhibition. J Dent Res. 93(10):1022-1027.

Wen X, Paine ML. 2013. Iron deposition and ferritin heavy chain (fth) localization in rodent teeth. BMC research notes. 6:1. 
Wurtz T, Houari S, Mauro N, MacDougall M, Peters H, Berdal A. 2008. Fluoride at non-toxic dose affects odontoblast gene expression in vitro. Toxicology. 249(1):26-34. 


\section{Figures legends}

\section{Figure 1. Iron detection in ameloblasts and enamel}

(A and C) Perl's staining during mouse amelogenesis shows iron content starting in early maturation- to pigmentation-stage ameloblasts. Chronic NaF exposure shortened the length of the blue-colored ameloblast-containing band, which also includes unstained cells (encircled in black). The bony ridge is marked with a dotted red line, allowing a comparison between each conditions, scale bar: $400 \mu \mathrm{m}$. (B and D) Higher magnification of ameloblasts colored by Perl's staining. Arrows (D) show groups of ameloblasts partially or completely devoid of staining or with unexpected iron accumulation when treated with $\mathrm{NaF}$. Scale bar: $50 \mu \mathrm{m}$. (E) Hemalun staining of $1 \mu \mathrm{m}$-thin frontal incisor sections for selection of regions of interest for SIMS imaging. Scale bar: $200 \mu \mathrm{m}$. (F) Iron content in mouse maturation-stage ameloblasts measured by SIMS imaging in a selected region. Iron, shown in red, was reduced in NaF-treated ameloblasts. Phosphorous, as internal control (in green), was mostly present in nuclear DNA and enamel. Am: ameloblasts, En: enamel, PL: papillary layer, scale bar: $30 \mu \mathrm{m}$. (G) Comparison of ESR spectra between control (C) and NaF-treated mouse incisors at room (293 K) and low (4.2 K) temperature. Under fluoride treatment, there was a significant decrease in intensity for the signals detected at $\mathrm{g} \approx 8.0, \mathrm{~g} \approx 2.4$, and $\mathrm{g}=2.06$, corresponding mainly to iron-bearing secondary phases (oxides and oxyhydroxides).

\section{Figure 2. NaF-induced expression modulations of mRNAs and proteins involved in iron} metabolism in the mouse enamel organ

(A) Relative levels of Hepcidin (Hamp), Transferin (Trf), Transferin receptor (Tfrc), Dmt-1 (Slc1 1a2), Fth, Ftl and Ferroportin (Slc40a1) mRNAs were measured in dental incisor epithelium 
by RT-qPCR. Relative expression levels were presented as ratios of test mRNA on reference RS15 mRNA (a.u., arbitrary units). (B-F) Immunofluorescent assays showing Fth staining in secretionstage (B) and in maturation-stage ameloblast cytoplasm (E) (in green) and iron transporters at the cell membranes, Tfrc (C), Slc11a2 (D) and Slc40a1 (F) (in red) in controls and NaF-treated mice. Nuclei were stained with DAPI (in blue). Am, ameloblast, PL: papillary layer, Scale bars $=30 \mu \mathrm{m}$.

(G and H) Western-blotting of whole mouse epithelial dental tissues showing Fth (G), Gapdh (used as the internal reference) and Slc40a1 (H). Means were calculated from the data of three independent experiments (each carried out on three different samples). Typical results are shown. Mean $\pm \mathrm{SD},(*) \mathrm{p} \leq 0.05,(* *) \mathrm{p} \leq 0.01$, ns: non-significant.

\section{Figure 3. Involvement of Fth in fluoride-induced enamel defects}

(A) Perl's staining showed reduced iron content in maturation-stage ameloblasts of $\mathrm{Fth}^{+/-}$mice. Exposure to NaF reduced the length of the stained ameloblast-containing band, with inclusion of uncolored ameloblast areas (encircled in black). The bony ridge is marked with a dotted red line, allowing comparison between samples. Scale bars: $400 \mu \mathrm{m}$. (B) Higher magnification of Perl's staining of maturation-stage ameloblasts. Scale bars: $50 \mu \mathrm{m}$. (C) Higher magnification of Perl's staining of pigmentation-stage ameloblasts. Scale bars: $50 \mu \mathrm{m}$. (D) Comparison of iron storage in maturation-stage ameloblasts of $\mathrm{Fth}^{+/}$, treated with $\mathrm{NaF}$ or not, and wild type mice by SIMS imaging. Iron content, shown in red, was strongly reduced in mature ameloblasts of $\mathrm{Fth}^{+/}$mice. Phosphorous used as internal control (in green), was mainly present in nuclear DNA and enamel. Am: ameloblasts, En: enamel, PL: papillary layer, scale bars: $30 \mu \mathrm{m}$. (E-H) Immunofluorescent assays using antibodies directed against mouse Tfrc (E), Slc11a2 (F), Fth (G) and Scl40a1 (H). Ap: apical side, Am: ameloblasts, PL: papillary layer, scale bar: $30 \mu \mathrm{m}$. (I) Relative levels of Fth 
measured by RT-qPCR in dental incisor epithelium of wild type and $\mathrm{Fth}^{+/-}$mice exposed or not to NaF. (J) Western-blotting of Fth and Gapdh. (K) Relative levels of Slc40a1 measured by RTqPCR in dental incisor epithelium of wild type and $\mathrm{Fth}^{+/-}$exposed or not to NaF. (L) Westernblotting of Slc40a1 and Gapdh. Means were calculated from the data of three independent experiments. Typical results are shown. Mean $\pm \mathrm{SD},(*) \mathrm{p} \leq 0.05,\left({ }^{*}\right) \mathrm{p} \leq 0.01,\left({ }^{* *}\right) \mathrm{p} \leq 0.001$, ns: non-significant.

\section{Figure 4. Structural and mechanical alterations of fluorotic teeth}

(A) Experimental fluorosis showing white opaque incisors of mice treated with $5 \mathrm{mM} \mathrm{NaF}$ (100 ppm) in the drinking water. (B) Opaque white incisors of $\mathrm{Fth}^{+/-}$mice and broken incisors of NaFtreated $\mathrm{Fth}^{+/-}$mice. (C) In mouse enamel incisor, all hardness values measured by nanoindentation were substantially reduced over the entire enamel thickness (red lines vs black lines) with the highest reductions in pigmented enamel. Hardness values in $\mathrm{Fth}^{+/-}$mouse enamel incisor decreased in pigmented enamel (dotted black lines) contrary to the situation recorded in wild type control mice (black lines). (D) Optical images and low-magnification SEM images of incisor frontal sections. Red arrows showed dark areas in outer enamel (OE) of $\mathrm{Fth}^{+/-}$mice. Scale bar: $500 \mu \mathrm{m}$. (E) SEM images showing the entire enamel thickness. OE: outer enamel, IE: inner enamel. Scale bar: $50 \mu \mathrm{m}$. (F) High-magnification SEM images of OE and IE with larger magnifications of rods with their scale bar are included (circled) in each image. R: rod, IR: interrod. Scale bar: $10 \mu \mathrm{m}$. (G) Enamel EDS measurements for comparison of $\mathrm{Ca} / \mathrm{P}$ ratios. Fth+/- enamel presented highly significant decreased $\mathrm{Ca} / \mathrm{P}$ ratio whatever the areas investigated $(\mathrm{n}=15$ measurements for each sample). Mean $\pm \mathrm{SD},(*) \mathrm{p} \leq 0.05,(* *) \mathrm{p} \leq 0.01,(* * *) \mathrm{p} \leq 0.001$ 
Figure 5. Schematic representation of the disruption of iron metabolism in the maturationstage enamel organ after chronic exposure to excessive $\mathrm{NaF}$

Under control conditions, $\mathrm{Fe}^{3+}$ transported in capillaries is distributed throughout the enamel organ by Trf and internalized by papillary cells and maturation-stage ameloblasts, essentially through Tfrc. Slc11a2 allows cell entry of $\mathrm{Fe}^{2+}$. Iron is stored in ameloblasts by Fth. Slc40a1 exports iron from ameloblasts apical side to the enamel matrix. Slc40a1 is also present at the basolateral membrane, where it exports iron to circulating blood. On the right, NaF reduces Fth expression in ameloblasts thus their iron content. In addition, available iron for secretion into the enamel matrix is further reduced because of apical Tfrc 're-uptake' and the decrease in Slc40a1 levels. The lack of iron that may occur during chronic exposure to fluoride results in enamel that is softer, less acidresistant, and uncolored. Resulting enamel defects may be used as a marker of iron-related disorders. 


\section{Figure 1}

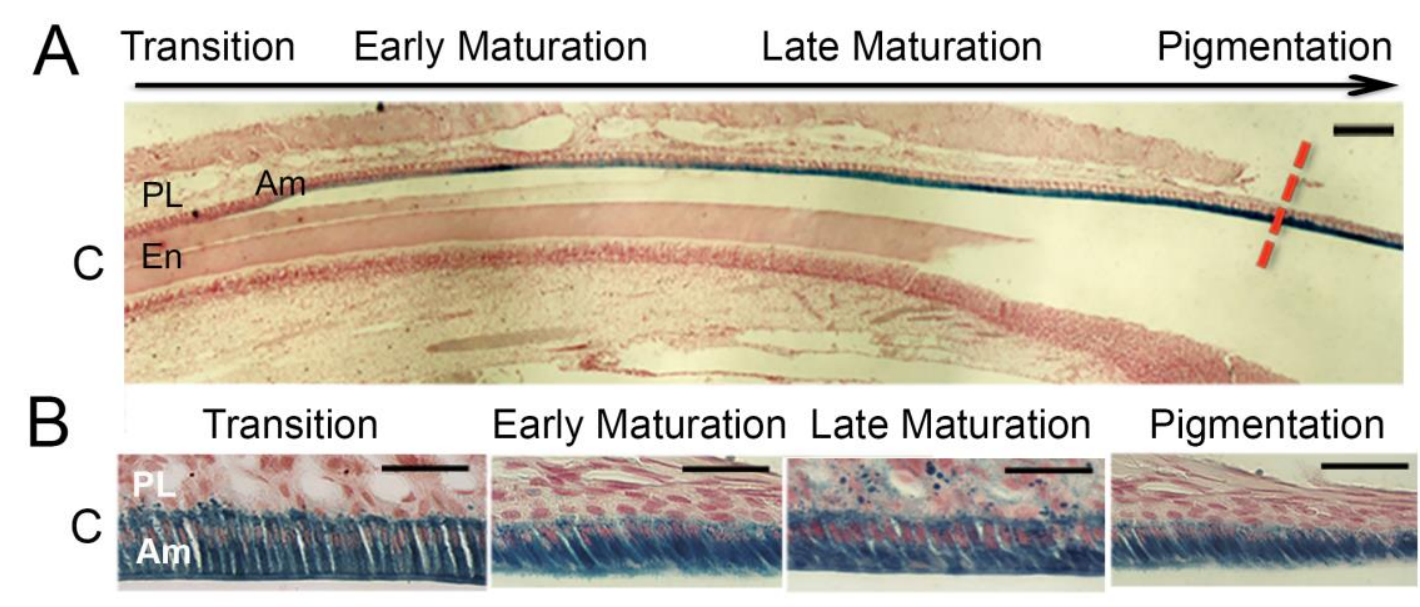

C Transition Early Maturation Late Maturation Pigmentation
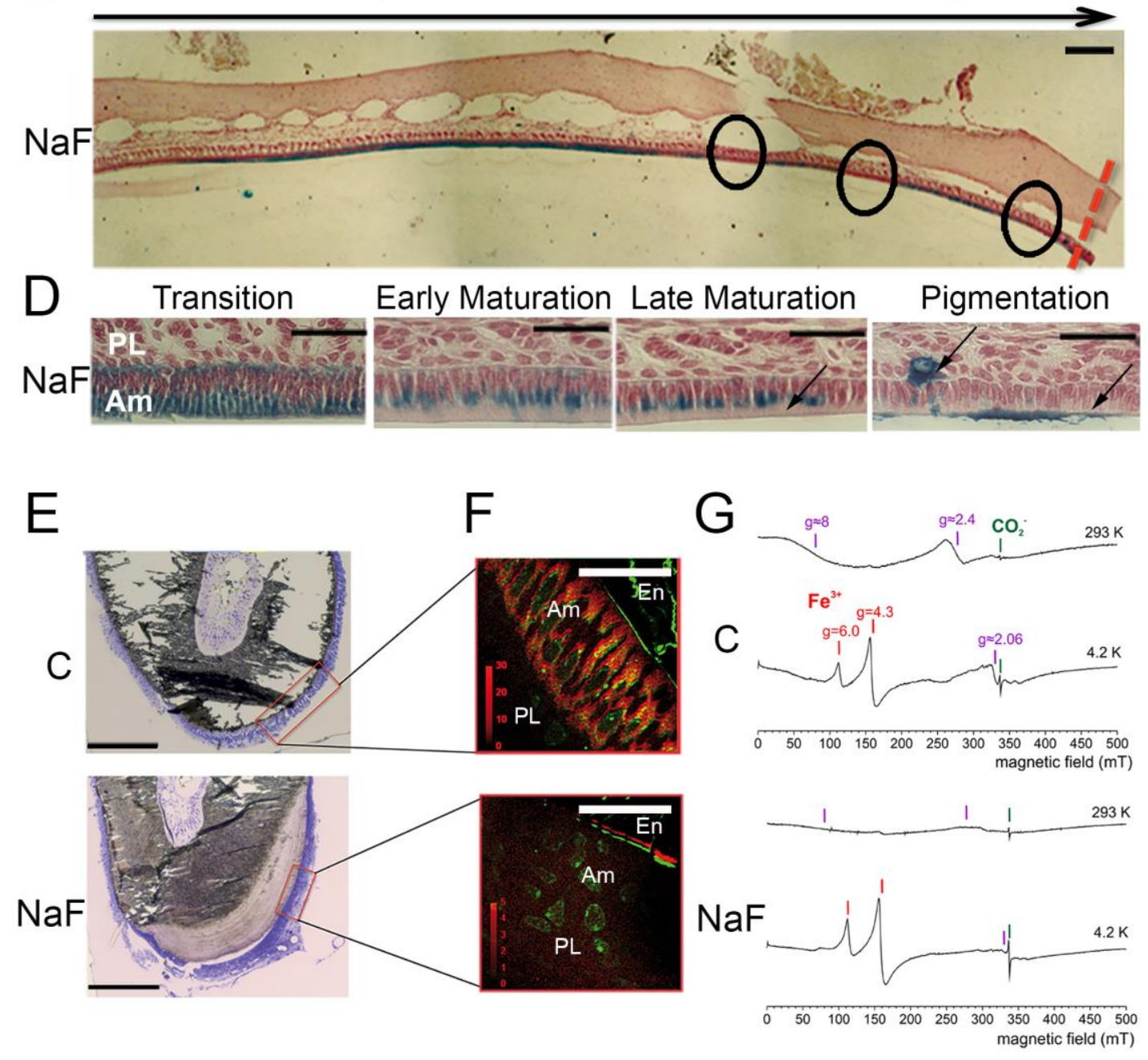
Figure 2
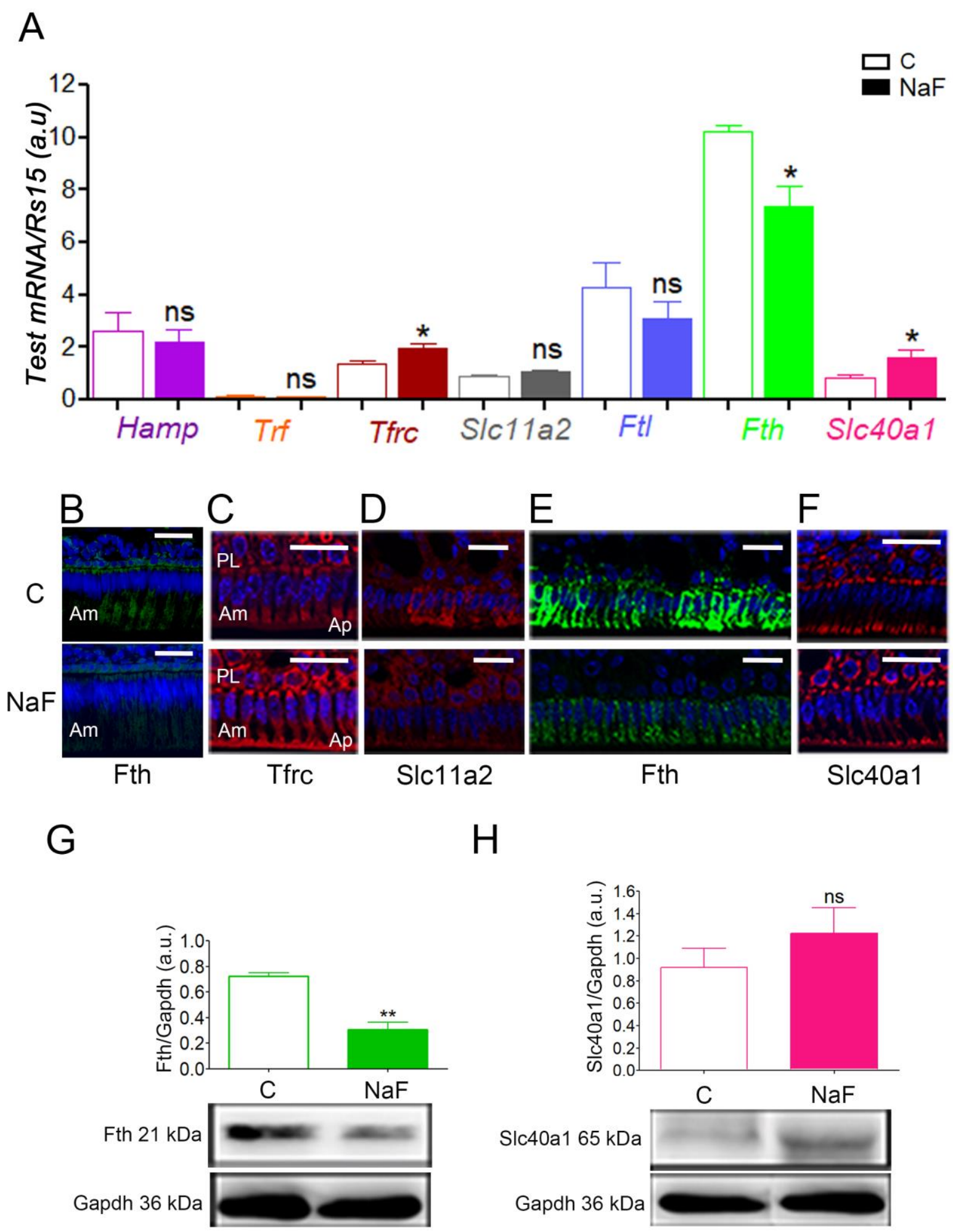


\section{Figure 3}
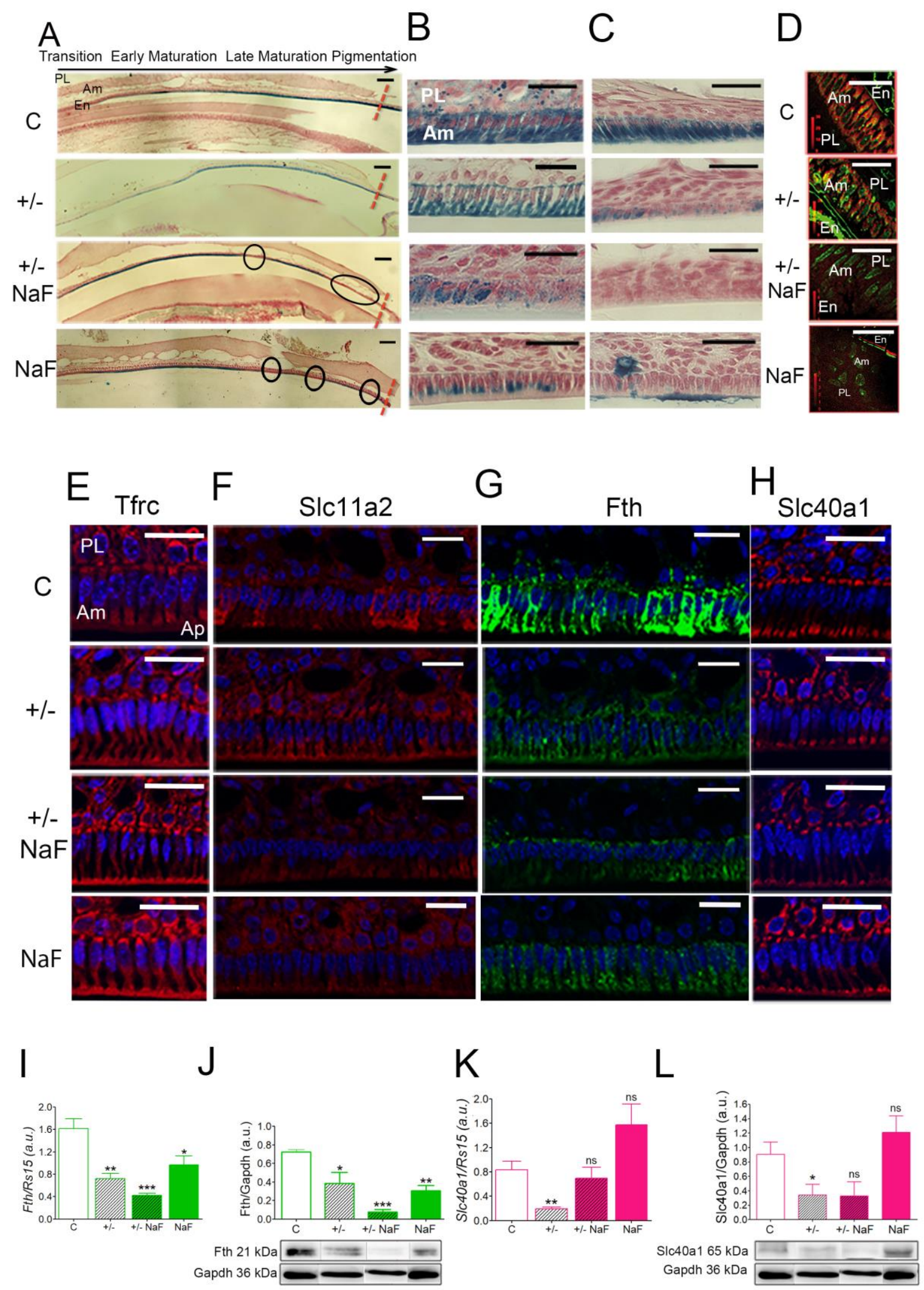
Figure 4
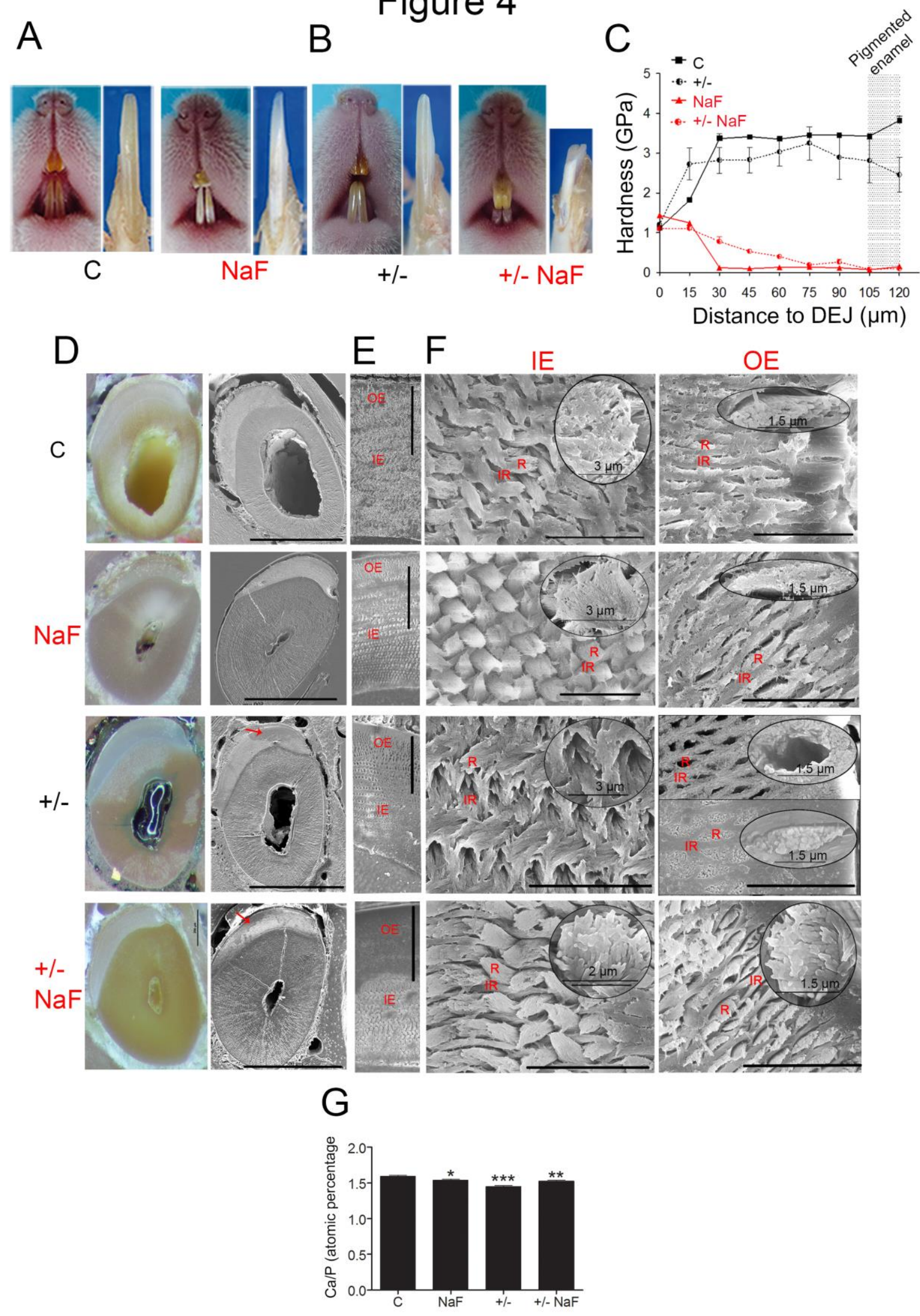


\section{Figure 5}

\section{Control}

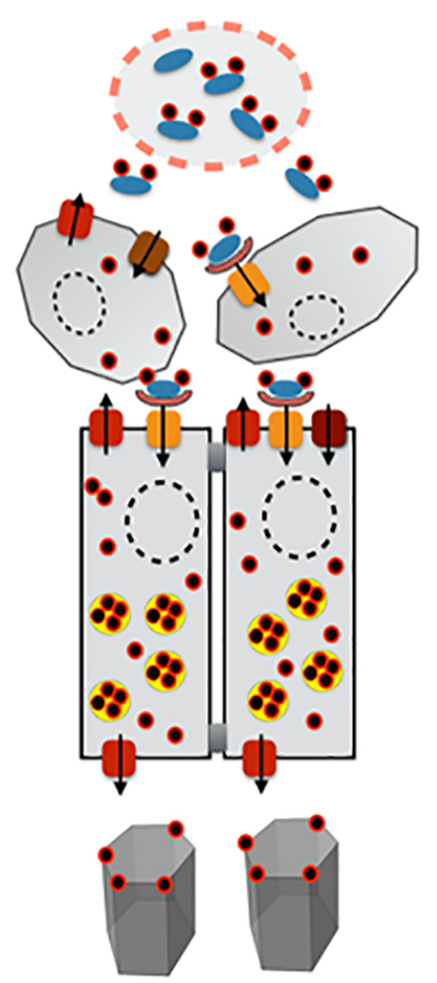

\section{NaF exposure}

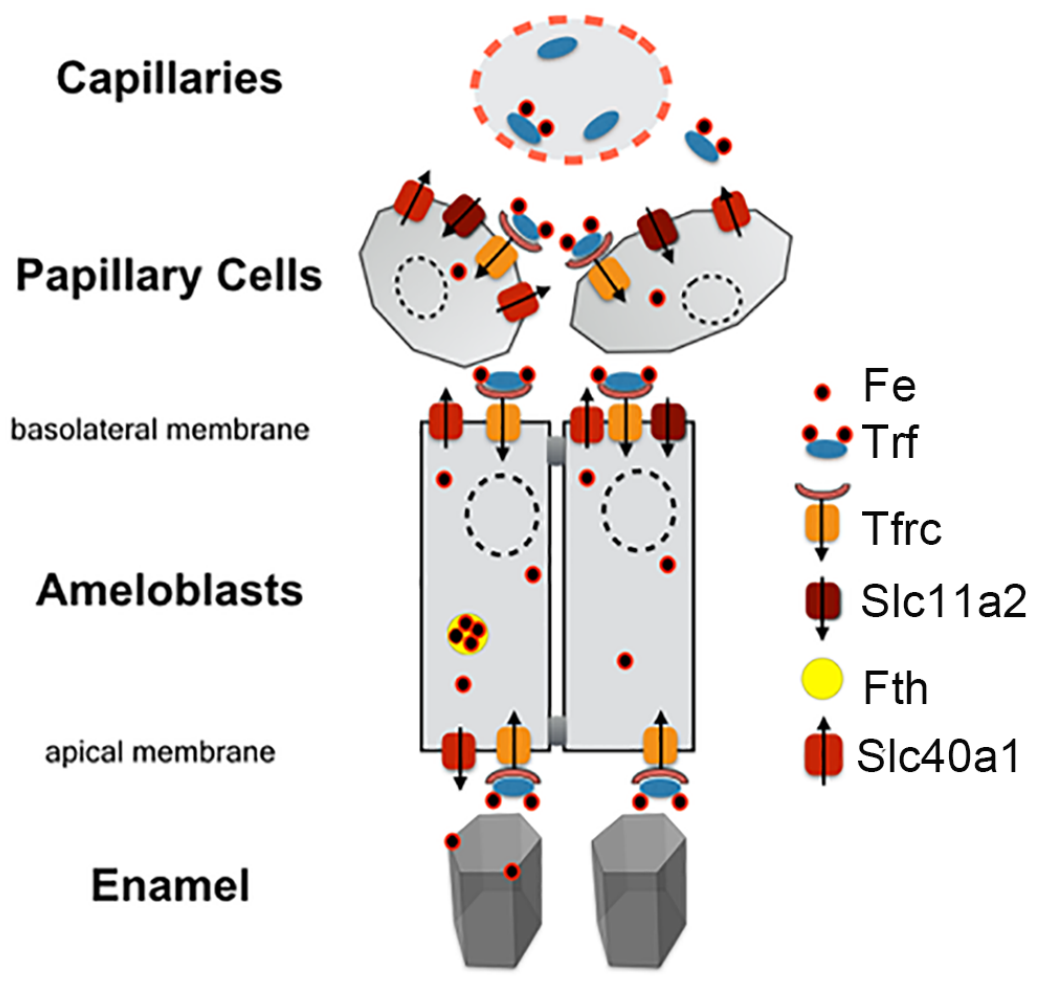

